OPEN ACCESS

Edited by: Barak Bar,

Loyola University Medical Center,

United States

Reviewed by:

Minjee Kim,

Northwestern University,

United States

Christoph Stretz,

Yale University, United States

${ }^{*}$ Correspondence:

Romain Gellens

romain.gellens@u-bordeaux.fr

Specialty section:

This article was submitted to

Neurocritical and

Neurohospitalist Care,

a section of the journal

Frontiers in Neurology

Received: 26 September 2017 Accepted: 20 December 2017

Published: 15 January 2018

Citation:

Gellens R, Habchi S, Freppel S, Couret D and lacobelli S (2018) Romiplostim for the Emergency

Management of Severe Immune

Thrombocytopenia with Intracerebral

Hemorrhage.

Front. Neurol. 8:737.

doi: 10.3389/fneur.2017.00737

\section{Romiplostim for the Emergency Management of Severe Immune Thrombocytopenia with Intracerebral Hemorrhage}

\author{
Romain Gellens ${ }^{1 *}$, Sabrina Habchi', Sebastien Freppel ${ }^{2}$, David Couret $^{1,3}$ and \\ Silvia lacobelli ${ }^{4,5}$
}

${ }^{1}$ Neurocritical Care Unit, Centre Hospitalier Universitaire Sud Réunion, Saint-Pierre, France, ${ }^{2}$ Department of Neurosurgery, Centre Hospitalier Universitaire Sud Réunion, Saint-Pierre, France, ${ }^{3}$ INSERM, UMR 1188 Diabète athérothrombose Thérapies Réunion Océan Indien (DéTROI), plateforme CYROI, Sainte Clotilde, France, ${ }^{4}$ Centre d'Études Périnatales de I'Océan Indien (CEPOI) - EA 7388, Centre Hospitalier Universitaire Sud Réunion, Saint-Pierre, France,

${ }^{5}$ Pediatric and neonatal intensive care unit, Centre Hospitalier Universitaire Sud Réunion, Saint-Pierre, France

Currently, we lack well-established guidelines for the emergency management of severe immune thrombocytopenia (ITP) with life-threatening bleeding. We now report the management of two patients with severe ITP, complicated by substantial cerebral hemorrhage, requiring urgent surgery due to refractory intracranial hypertension. To rapidly boost platelet counts (PCs), corticosteroids, intravenous immunoglobulin, and iterative platelet transfusions were given; all were ineffectual. Romiplostim, a thrombopoietin receptor agonist, was then administered as an "on demand therapy," with the result that a rapid and sustained increase of PCs was achieved, thus allowing for postoperative hemostasis. Both patients recovered good neurological condition, suggesting the potential utility of romiplostim, in combined therapy, for the emergency management of severe ITP.

Keywords: immune thrombocytopenia, romiplostim, thrombopoietin receptor agonist, intracerebral hemorrhage, hemorrhagic stroke

\section{INTRODUCTION}

Intracerebral hemorrhage ( $\mathrm{ICH})$ is a rare $(1.4 \%$ in adults, $<1 \%$ in children) (1) but devastating complication of severe immune thrombocytopenia (ITP), with high mortality (up to 25\%) and morbidity ( $25 \%$ of neurologic sequelae) (2). Currently, we lack well-established guidelines for the emergency management of severe ITP with ICH.

In 2009, an international working group (3) proposed a new definition of immune thrombocytopenic purpura, adjusted in 2011 by The American Society of Hematology (4). ITP, as newly defined, is an acquired disease characterized by a platelet count (PC) less than $100 \times 10^{9} / \mathrm{L}$ mediated by immune system disruption. In primary ITP, thrombocytopenia is isolated and unrelated to any obvious etiology. Secondary ITP is considered to be the result of underlying disease (infection, autoimmunity, and neoplastic disorder), or drug exposure, and its evolution often correlates with management of the triggering factor. ITP can be divided into three stages: newly diagnosed ( $0-3$ months), persistent (3-12 months duration), and chronic ITP (lasting $>12$ months). The degree of the severity of ITP should be assessed by bleeding symptoms rather than by PC. However, ITP-specific bleeding measurement tools are not yet validated by learned societies (1). In guidelines and consensus reports (3, 4 ), the term "severe ITP" is reserved for patients who have clinically relevant bleeding; that is to say 
a bleeding considered sufficient in magnitude either to mandate a treatment or which requires additional intervention, regardless of the PC.

In adults, treatment of ITP should only be considered if there is a high risk of bleeding, commonly defined by a $\mathrm{PC}<30 \times 10(9) / \mathrm{L}$ or in the case of severe ITP (3-5). First-line treatment consists of corticosteroids and/or intravenous immunoglobulin (IVIg), effective in $70-90 \%$ of patients, with median times to response of 4-14 and 1-4 days, respectively (5). Patients not responding to the first-line treatment require second-line therapy including splenectomy or various immunosuppressive agents that are not always appropriate for critically ill. Therefore, "on demand therapy" designates any therapy used to temporarily increase the PC in cases of major bleeding or to safely perform invasive procedures $(3,5)$.

Immune thrombocytopenia pathogenesis is mediated by autoantibodies resulting in both accelerated circulating platelet destruction and impaired platelet production. The second mechanism has prompted the recent development of drugs such as thrombopoietin (TPO) growth factor, and, latterly, TPO-receptor agonists (6). Romiplostim is a second-generation TPO-R agonist whose efficacy and safety have been assessed in recent randomized trials, especially in chronic $\operatorname{ITP}(7,8)$. The US Food and Drug Administration and the European Medicine Agency have approved romiplostim for adults with chronic ITP who are unresponsive to corticosteroids, IVIg, or splenectomy. The recent ITP management guidelines $(4,5)$ also recommend or suggest TPO-R agonists as a second-line therapy for adults at risk of bleeding regardless of their splenectomized status: romiplostim showing an $89 \%$ response rate in nonsplenectomized patients (8). Neither guideline recommends the use of romiplostim in children because of their common, spontaneous, and rapid remission. However, several recent randomized trials in children support the safety and efficacy of romiplostim in chronic ITP, with an overall response rate comparable to adults (9).

Romiplostim was also proposed in the preoperative period of elective surgeries (10), and as "on demand therapy" for patients with severe mucocutaneous bleeding, in newly diagnosed or chronic ITP $(11,12)$. However, there are very few reports of its use in the setting of ICH. We now describe two cases (one adult and one child) illustrating the use of romiplostim as "on demand therapy" for hemorrhagic stroke causing intracranial hypertension, in the setting of primary or secondary ITP. We consider a $\mathrm{PC}>100 \times 10^{9} / \mathrm{L}$ as a complete response to treatment, which is also the target PC commonly accepted for ICH. We obtained written and informed consent from the participants to publish this report.

\section{CASE 1}

A 29-year-old man, without a past medical history, was admitted in September 2015 at Réunion University Hospital for sepsis with eosinophilic pneumonia (Figures 1 and 2). His blood cell count revealed severe thrombocytopenia $\left(31 \times 10^{9} / \mathrm{L}\right)$ with hypereosinophilia $\left(8 \times 10^{9} / \mathrm{L}\right)$. Amoxicillin/clavulanic acid had been prescribed 1 week earlier for an inflammatory axillary tumefaction that occurred after shaving. The initial diagnostic workup revealed only pulmonary disorders: bronchoalveolar lavage showing marked eosinophilia (97\%), alveolar hemorrhage, with quantitative culture of $10^{4} \mathrm{CFU} / \mathrm{mL}$ Streptococcus oralis. A bone marrow aspirate revealed rich marrow proliferation. Screens for

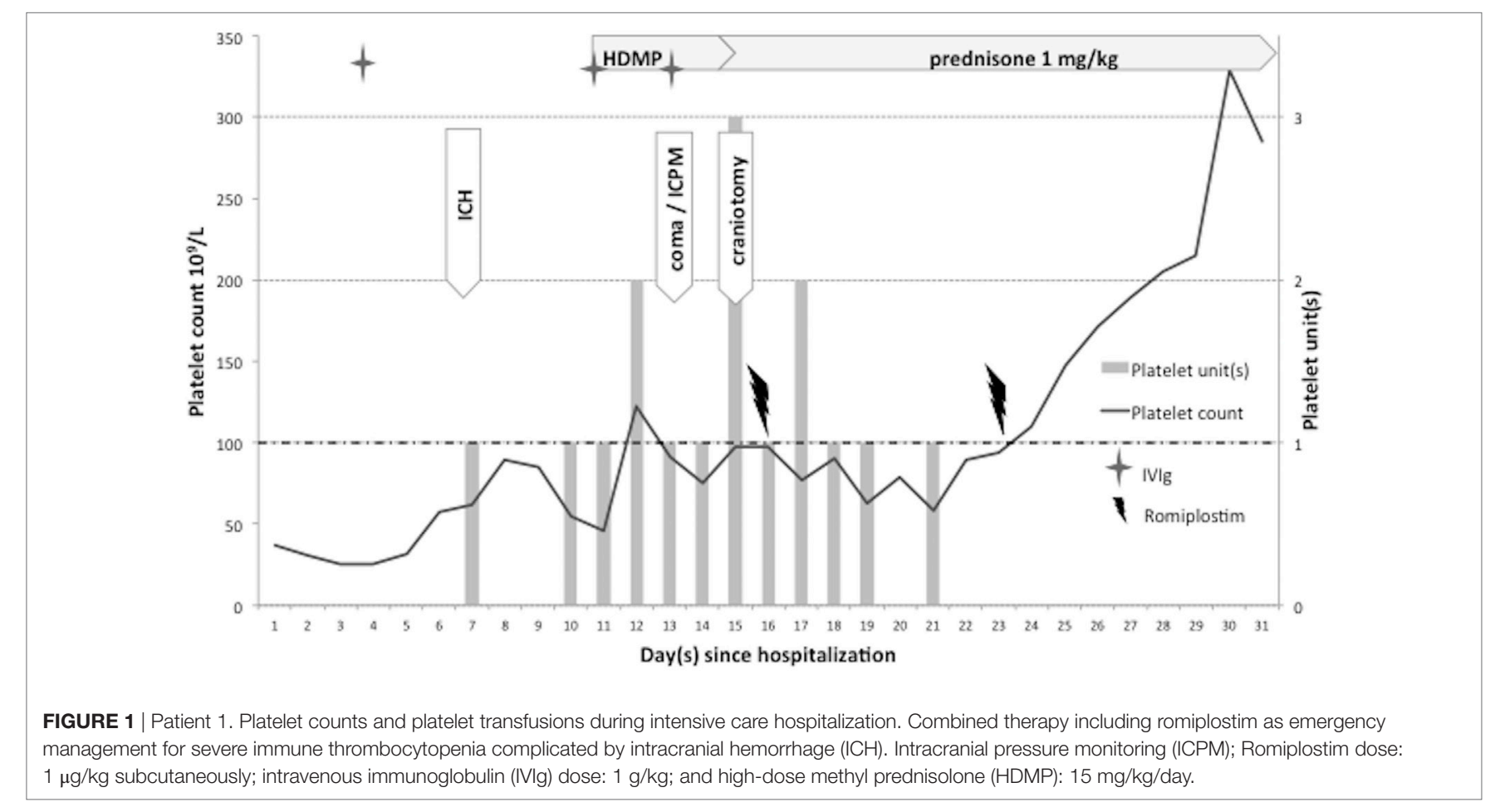




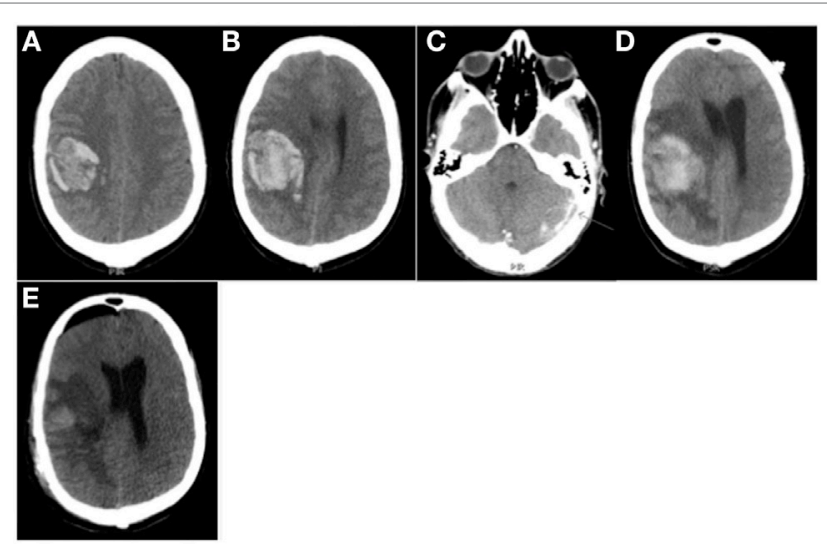

FIGURE 2 | Patient 1. CT head (A,B,D) and CT venography (C) prior to surgery: (A) Day 6: large right frontal cerebral hematoma (40 $\mathrm{mL})$, moderate mass effect with $4 \mathrm{~mm}$ of maximal brain midline shift. (B) Day 7: hematoma volume increase up to $45 \mathrm{~mL}$. (C) Day 13: left lateral venous sinus thrombosis. (D) Day 14, just before surgery: increased mass effect $(10 \mathrm{~mm}$ brain midline shift) with peripheral edema. CT head after surgery: (E) Day 16: decreased mass effect (6 $\mathrm{mm}$ brain midline shift).

autoimmune disease (including anti-phospholipids and ANCA vasculitis test) were negative; no allergies were reported. In the context of sepsis and a probable diagnosis of secondary ITP, the patient received a first course of IVIg, combined with antibiotics, and antiparasitics.

Six days after admission, the patient was transferred to the neurointensive care unit for both spontaneous intracerebral and abdominal hemorrhage. He presented with drowsiness, predominantly brachiofacial hemiplegia associated with lower back pain, and a PC of $60 \times 10^{9} / \mathrm{L}$. A CT scan showed a large hemorrhage in the right frontal lobe $(40 \mathrm{~mL})$ with mass effect (Figure 2A) and a large $(6 \mathrm{~cm})$ left adrenal gland hematoma. CT angiography revealed a spot sign within the brain hematoma without any suggestion of arteriovenous malformation or cerebral venous thrombosis. A 24-h follow-up CT scan revealed a slight expansion of the frontal hematoma ( 45 vs. $40 \mathrm{~mL}$ ) (Figure 2B). Therefore, ITP therapy was intensified with iterative platelet transfusions to achieve a $\mathrm{PC} \geq 100 \times 10^{9} / \mathrm{L}$.

At day 12, the patient was found to be in convulsive status epilepticus, and clinical seizure control was achieved with antiepileptic medication (benzodiazepine, then phenytoin). A sustainable decrease in Glasgow Coma Score to 8 required general anesthesia with IV midazolam and mechanical ventilation. An EEG revealed a non-convulsive status epilepticus controlled with propofol infusion. A brain CT venography showed a left lateral sinus thrombosis complicated by small temporal hemorrhage (Figure 2C). An intraparenchymal pressure monitor (Codman ${ }^{\circledR}$ MicroSensor) was placed in the left frontal lobe; revealing an initial intracranial pressure (ICP) of $40 \mathrm{mmHg}$. ICP decreased only transiently despite deeper level of standard sedation (propofol, midazolam, and sufentanil), prevention of secondary systemic brain insults, pharmacological neuromuscular paralysis, and controlled mild hypothermia $\left(35^{\circ} \mathrm{C}\right)$. Finally, a barbiturate coma was required to control intracranial hypertension but ICP increased again above
$40 \mathrm{mmHg}$ at day 14. A new CT scan showed no expansion of the right frontal hematoma but substantial worsening of edema with increased mass effect (Figure 2D). Because of refractory intracranial hypertension (ICHT), a mini-craniotomy was performed to evacuate the hematoma, although iterative platelet transfusions failed to secure the procedure and avoid perioperative bleeding. Thus, romiplostim was initiated, at the advice of the hematologist, with a first injection the day after surgery and a second course 1 week later. A sustainable complete response was achieved 8 days after first administering romiplostim. No further hemorrhage expansion or new bleeding into the craniotomy site was noted on follow-up brain imaging (Figure 2E). Note that immunosuppressive agents could not be used because of a concomitant septic shock related to a pyothorax.

Heparin could then be initiated for cerebral sinus thrombosis and further invasive procedures performed to complete the diagnostic workup (lung biopsy, transesophageal echocardiography, and cerebral angiogram), which remained non-contributory. Thereafter, the patient was discharged from hospital 2 months after being admitted with predominantly brachiofacial paresis.

\section{CASE 2}

A 14-year-old female teenager, with a previous medical history of chronic ITP (diagnosed in 2012), was admitted to Mayotte's Hospital (French overseas department) in October 2015 for gingival bleeding, intense headaches, and drowsiness (Figures 3 and 4). A blood cell count revealed severe thrombocytopenia at $13 \times 10^{9} / \mathrm{L}$ and hemoglobin of $80 \mathrm{~g} / \mathrm{L}$. A CT scan showed a $36 \mathrm{~mL}$ right parieto-occipital hematoma with significant perilesional edema and mass effect (Figure 4A). She received first-line therapy including corticosteroids, IVIg, and platelet transfusion. Subsequently, she had a generalized tonic-clonic seizure. On the third day, she was transferred to the Pediatric Intensive Care Unit of Saint Pierre (Reunion Island, France), which is equipped with a neurosurgical department.

After admission, the neurological status of the patient deteriorated, with coma and anisocoria related to cerebral hematoma growth $(50 \mathrm{~mL})$ (Figure 4B). A barbiturate-induced coma was required to control ICHT, and corticotherapy was intensified because of persistent severe thrombocytopenia. The day after, ICHT remained uncontrolled. Salvage surgery was performed (craniotomy with hematoma evacuation). Despite massive platelet transfusion in the perioperative period, the $\mathrm{PC}$ remained below $15 \times 10^{9} / \mathrm{L}$ before the procedure, which was complicated by significant subcutaneous bleeding (Figure 4C). Consequently, romiplostim was initiated at the advice of the hematologist, with a first administration the day after surgery and a second course 1 week later. At this point, a PC of $81 \times 10^{9} / \mathrm{L}$ was achieved, largely due to a massive platelet transfusion. A sustainable "complete response" was achieved only 6 days after romiplostim administration. We noted a PC peak of $1,554 \times 10^{9} / \mathrm{L} 2$ weeks after the second romiplostim injection, without clinical consequences. No further hemorrhage expansion or new bleeding into the craniotomy site was noted on follow-up brain imaging (Figure 4D). No hemorrhagic complication was observed related to the intraparenchymal ICP monitor (Figure 4E) placed at the end of 


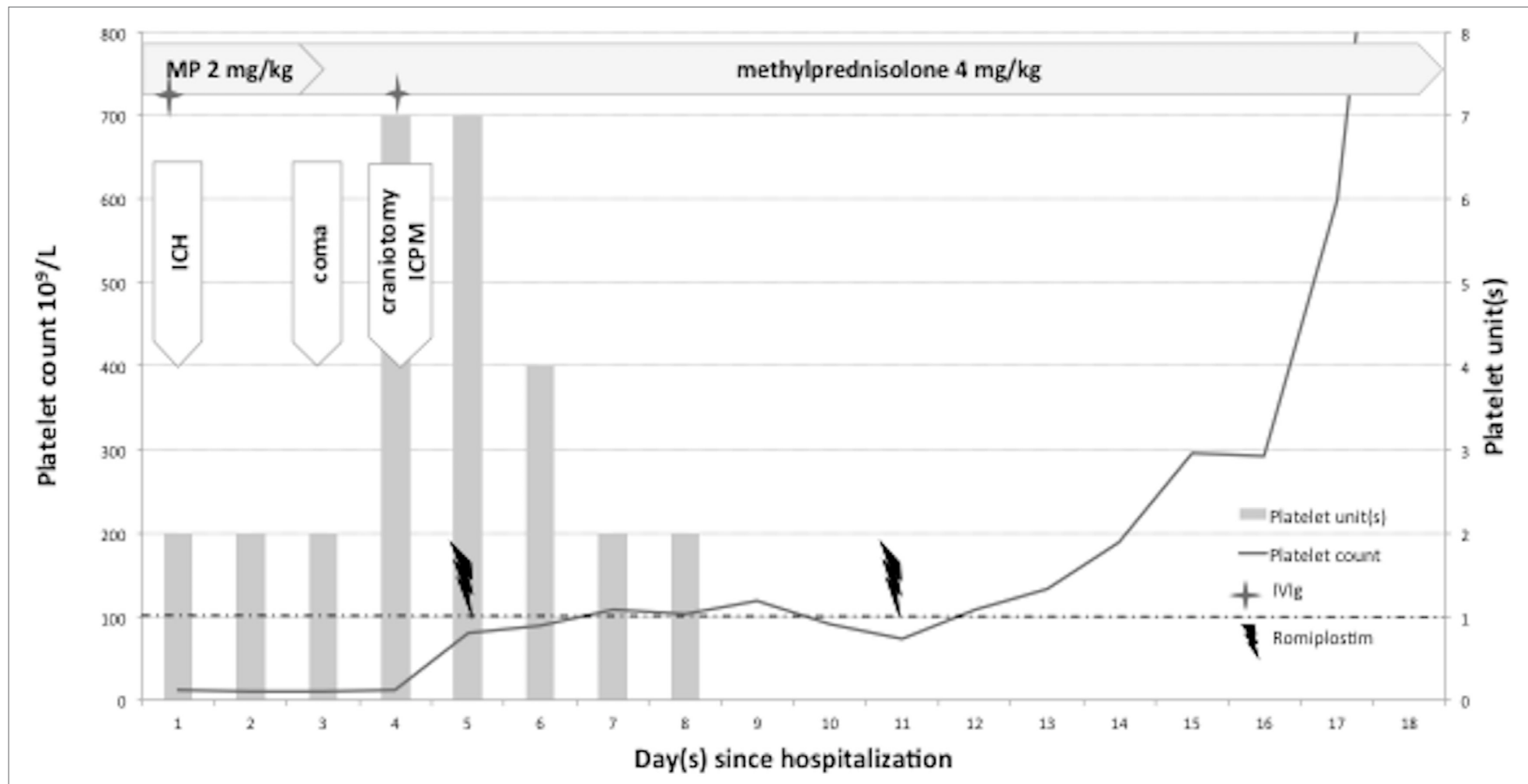

FIGURE 3 | Patient 2. Platelet counts and platelet transfusions during intensive care hospitalization. Combined therapy including romiplostim as emergency management for severe ITP complicated by intracranial hemorrhage $(\mathrm{ICH})$. ICPM = intracranial pressure monitoring (intraparenchymal probe). MP = methylprednisolone. Romiplostim dose: $10 \mu \mathrm{g} / \mathrm{kg}$ subcutaneously; intravenous immunoglobulin (IVIg) dose: $1 \mathrm{~g} / \mathrm{kg}$.

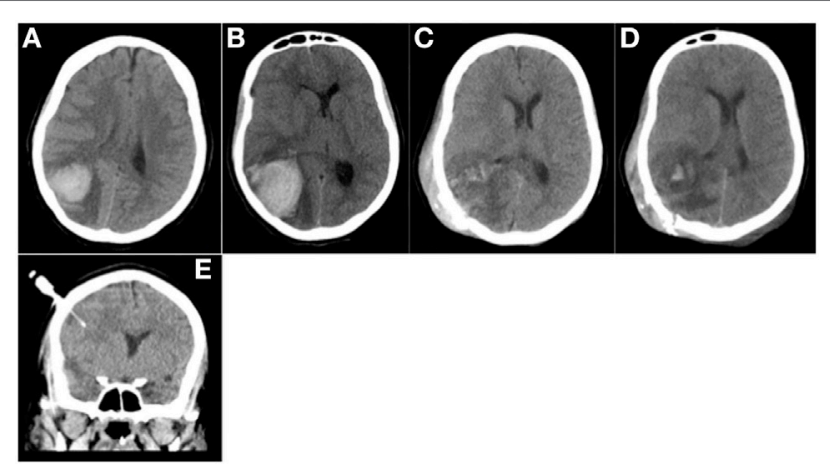

FIGURE 4 | Patient 2. CT head (A,B) prior to surgery: (A) Day 1: $36 \mathrm{~mL}$ parieto-occipital hematoma with perilesional edema and slight mass effect. (B) Day 3: significant hematoma expansion (hematoma volume measured at $50 \mathrm{~mL}$ ) and increased mass effect. CT head (C-E) after surgery: (C) Day 5; the day after surgery: hematoma evacuation; decreased mass effect; subcutaneous hematoma occurring during surgery. (D) Day 10: no rebleeding was noted. (E) Day 10: no hemorrhage was observed around the intraparenchymal probe (intracranial pressure monitoring).

the surgical procedure. ICP was initially measured at $27 \mathrm{mmHg}$ and then decreased gradually by continuing sedation for a few more days.

Eighteen days after her hospitalization the patient was discharged from intensive care with left residual hemiparesis. She full recovered a few months later.

\section{DISCUSSION}

Hematoma volume and hematoma growth are key determinants of poor outcomes for ICH caused by hematologic disorders (13). Therefore, the aim of emergent management of ITP in the setting of $\mathrm{ICH}$ is to achieve a rapid and sufficient PC to stop bleeding, avoid hematoma growth, and permit operative intervention. Combination therapy (IVIg associated with corticosteroids and iterative platelet transfusions) appears to provide the most rapid rise in PC $(4,5)$ but may not be enough, especially in the setting of ICH where high PC level is required $\left(80-100 \times 10^{9} / \mathrm{L}\right)$, which leads to massive platelet transfusion. Critical illness (ICHT, sepsis) often limits the use of second-line ITP therapy. Our cases support the efficacy and safety of the emergency use of romiplostim to promptly restore a safe PC and secure the postoperative period in the setting of unresponsive and severe ITP with ICH. A sustainable complete PC response was obtained for both patients with a short course of romiplostim, and platelet transfusions were rapidly stopped. No significant side effects related to romiplostim (14) were reported despite many confounding factors due to the critical illness: the thrombosis in case 1 pre-existed romiplostim administration, with no rebound of deteriorating thrombocytopenia following romiplostim cessation.

The shorter time to response noted in our experience using romiplostim to achieve a $\mathrm{PC}>100 \times 10^{9} / \mathrm{L}$ compared to that reported in the Contis study (11) ( $\sim 7$ days vs. $\sim 2$ weeks) may be explained by our early initiation of romiplostim treatment after 
corticosteroids and IVIg ( $<7$ days vs. several weeks). Given the need to reduce the time to response of ITP treatment to prevent cerebral hematoma growth, romiplostim could be associated with the combined first-line therapy for ITP patients with ICH and as much as possible before surgical procedure.

In the setting of combined ITP therapy, our experience suggests that timely neurosurgical intervention appears to be an acceptable risk as previously reported (15). Note that none of our patients experienced hemorrhagic complications after intracranial pressure monitor insertion (intraparenchymal probes were placed after platelet transfusion).

The doses used in our cases are those usually recommended in the literature but correspond to extreme values (range from 1 to $10 \mu \mathrm{g} / \mathrm{kg}$ weekly) (6-9). Indeed, the usual recommended starting dose is 3-6 $\mu \mathrm{g} / \mathrm{kg} /$ weekly. The intensity of the PC response appears to be related to a "dose effect" of romiplostim as previously reported (6).

Significant sparing of platelet transfusions contributes to the cost-effectiveness of romiplostim (16). Indeed, to achieve a PC of $100 \times 10^{9} / \mathrm{L}$, our patients received 16 and 28 platelet concentrates, the majority before romiplostim administration. In fact, previous work suggests that romiplostim leads to bleeding cessation before the PC increases. This could be related to an activation of pre-existing platelets and a restoration of immune tolerance to platelet antigens (14).

\section{REFERENCES}

1. Neunert C, Noroozi N, Norman G, Buchanan GR, Goy J, Nazi I, et al. Severe bleeding events in adults and children with primary immune thrombocytopenia: a systematic review. J Thromb Haemost (2015) 13(3):457-64. doi:10.1111/ jth.13019

2. Psaila B, Petrovic A, Page LK, Menell J, Schonholz M, Bussel JB. Intracranial hemorrhage (ICH) in children with immune thrombocytopenia (ITP): study of 40 cases. Blood (2009) 114(23):4777-83. doi:10.1182/blood-2009-04-215525

3. Rodeghiero F, Stasi R, Gernsheimer T, Michel M, Provan D, Arnold DM, et al. Standardization of terminology, definitions and outcome criteria in immune thrombocytopenic purpura of adults and children: report from an international working group. Blood (2009) 113(11):2386-93. doi:10.1182/ blood-2008-07-162503

4. Neunert C, Lim W, Crowther M, Cohen A, Solberg L, Crowther MA, et al. The American Society of Hematology 2011 evidence-based practice guideline for immune thrombocytopenia. Blood (2011) 117(16):4190-207. doi:10.1182/ blood-2010-08-302984

5. Provan D, Stasi R, Newland AC, Blanchette VS, Bolton-Maggs P, Bussel JB, et al. International consensus report on the investigation and management of primary immune thrombocytopenia. Blood (2010) 115(2):168-86. doi:10.1182/blood-2009-06-225565

6. Bussel JB, Kuter DJ, George JN, McMillan R, Aledort LM, Conklin GT, et al. AMG 531, a thrombopoiesis-stimulating protein, for chronic ITP. N Engl $J$ Med (2006) 355(16):1672-81. doi:10.1056/NEJMoa054626

7. Kuter DJ, Bussel JB, Lyons RM, Pullarkat V, Gernsheimer TB, Senecal FM, et al. Efficacy of romiplostim in patients with chronic immune thrombocytopenic purpura: a double-blind randomised controlled trial. Lancet (2008) 371(9610):395-403. doi:10.1016/S0140-6736(08)60203-2

8. Kuter DJ, Rummel M, Boccia R, Macik BG, Pabinger I, Selleslag D, et al. Romiplostim or standard of care in patients with immune thrombocytopenia. N Engl J Med (2010) 363(20):1889-99. doi:10.1056/NEJMoa1002625

9. Bussel JB, Buchanan GR, Nugent DJ, Gnarra DJ, Bomgaars LR, Blanchette VS, et al. A randomized, double-blind study of romiplostim to determine its safety and efficacy in children with immune thrombocytopenia. Blood (2011) 118(1):28-36. doi:10.1182/blood-2010-10-313908
In conclusion, our cases support the potential utility of a combined therapy including romiplostim, for the emergent management of ICH in the setting of primary or secondary ITP in adults and pediatric patients.

\section{ETHICS STATEMENT}

Case report: patient consents are in attached files.

\section{AUTHOR CONTRIBUTIONS}

RG: conception and design, acquisition of data, drafting the article, reviewed submitted version of manuscript, approved the final version of the manuscript on behalf of all authors, administrative/ technical/material support, and study supervision. SH: conception and design, drafting the article, critically revising the article, reviewed submitted version of manuscript, and study supervision. SF: critically revising the article and reviewed submitted version of manuscript. DC: conception and design, critically revising the article, reviewed submitted version of manuscript, approved the final version of the manuscript, and study supervision. SI: conception and design, acquisition of data, critically revising the article, reviewed submitted version of manuscript, approved the final version of the manuscript, administrative/technical/material support and study supervision.

10. Ramakrishna R, Rehman A, Ramakrishna S, Alexander W, Yeo WW. Use of romiplostim in patients with chronic idiopathic thrombocytopenic purpura during perioperative period. Intern Med J (2015) 45(7):718-24. doi:10.1111/ imj.12794

11. Contis A, Lazaro E, Greib C, Pellegrin J-L, Viallard J-F. Romiplostim as early treatment for refractory primary immune thrombocytopenia. Int J Hematol (2013) 98(5):520-4. doi:10.1007/s12185-013-1439-0

12. Sivera P, Ruella M, Gueli A, Hu H, Wade M, Tarella C. Use of the novel thrombopoietin receptor-agonist romiplostim, in combination with steroids and immunoglobulins for the increase of platelets prior to splenectomy, in refractory immune thrombocytopenia: a case report. Blood Coagul Fibrinolysis (2012) 23(4):331-4. doi:10.1097/MBC.0b013e3283513a97

13. Brouwers HB, Greenberg SM. Hematoma expansion following acute intracerebral hemorrhage. Cerebrovasc Dis (2013) 35(3):195-201. doi:10.1159/000346599

14. Chalmers S, Tarantino MD. Romiplostim as a treatment for immune thrombocytopenia: a review. J Blood Med (2015) 6:37-44. doi:10.2147/ JBM.S47240

15. So MY, Li CH, Lee ACW, Kwong NS. Intracranial haemorrhage among Chinese children with immune thrombocytopenia in a Hong Kong regional hospital. Hong Kong Med J (2013) 19(2):129-34.

16. Lee D, Thornton P, Hirst A, Kutikova L, Deuson R, Brereton N. Cost effectiveness of romiplostim for the treatment of chronic immune thrombocytopenia in Ireland. Appl Health Econ Health Policy (2013) 11(5):457-69. doi:10.1007/ s40258-013-0044-y

Conflict of Interest Statement: The authors declare that the research was conducted in the absence of any commercial or financial relationships that could be construed as a potential conflict of interest.

Copyright (c) 2018 Gellens, Habchi, Freppel, Couret and Iacobelli. This is an open-access article distributed under the terms of the Creative Commons Attribution License (CC BY). The use, distribution or reproduction in other forums is permitted, provided the original author(s) or licensor are credited and that the original publication in this journal is cited, in accordance with accepted academic practice. No use, distribution or reproduction is permitted which does not comply with these terms. 